\title{
Androgen receptor and its splice variants in prostate cancer
}

\author{
Simon Haile and Marianne D. Sadar ${ }^{*}$ \\ Genome Sciences Centre, BC Cancer Agency, 675 west $10^{\text {th }}$ Avenue, Vancouver, BC, Canada \\ V5Z 1 L3
}

\begin{abstract}
Androgen receptor (AR) is a transcription factor that becomes active upon binding to androgens via its ligand-binding domain (LBD) or in response to signaling cascades initiated by growth factors and cytokines. The activity of AR requires regions within the $\mathrm{N}$-terminal domain (NTD) in a manner that is distinct from the activation of related steroid hormone receptors. Unequivocal evidence has been amassed to consider that the AR axis is the most critical pathway for the progression of prostate cancer. Qualitatively distinct insight into AR pathobiology has been garnered that include AR-regulated gene expression is stage-specifically modulated during disease progression and that ligand requirement for AR activity could be rendered dispensable due to expression of constitutively active AR splice variants that are devoid of LBD. The recent appreciation of the clinical challenge that stems from non-gonadal androgens that are not inhibited by traditional hormonal therapies has been tangibly translated into the development of more potent drugs that can potentially lead towards achieving an androgen-free environment. The pre-clinical evidence that proves the AR NTD is a druggable target also forecasts further paradigm shift in the management of advanced prostate cancer. These advancements together with the identification of more robust AR antagonists and their promising clinical outcome has renewed the hope that targeting the AR pathway remains a sound strategy in the clinical management of prostate cancer. Here, we address these developments with a greater emphasis to the rapidly growing literature on AR splice variants.
\end{abstract}

\section{Keywords}

Androgen receptor; splice variants; castration-resistant prostate cancer; CRPC; NTD; aminoterminus; LBD; prostate cancer

\section{Prostate Cancer}

Prostate cancer remains one of the most diagnosed and lethal cancers for men in the Western world. Localized disease is treatable and potentially curable with surgery or radiation therapy. However, the disease will recur in $20-40 \%$ of patients following these treatments. Patients with recurrent disease and those who have systemic disease have poor prognosis. The first line of treatment for recurrent and/or systemic disease is surgical or chemical castration and the application of anti-androgens (Figure 1). While there is initial response to androgen ablation treatments, most patients unfortunately develop resistance, at which stage the disease is referred to as castration-resistant prostate cancer (CRPC). Current treatments available for CRPC include taxanes and Sipuleucel-T that increase survival by only a few months. Most patients succumb to CRPC within 2 years of onset emphasizing the urgent need to develop treatment options for this lethal form of the disease.

\footnotetext{
"Correspondence to: Dr. Marianne D. Sadar; msadar@bcgsc.ca; Phone: 604-675 8157; FAX: 604-675 8178.
} 


\section{Androgen Receptor as a Validated Target for CRPC}

Androgen receptor (AR) is essential for the growth of CRPC in vitro and in xenograft models [1]. AR is a type I nuclear receptor that mediates the effects of androgens such as testosterone and dihydrotestosterone (DHT). The human AR gene is located on chromosome Xq11-12 and spans $\sim 180 \mathrm{~kb}$ of DNA containing 8 canonical exons. Recently, novel exons that map within introns flanking the canonical exons as well as an additional exon that extends the $3^{\prime}$ untranslated region have been described [2, 3] (Figure 2; Table 1).

Androgen binds to the $\mathrm{COOH}$-terminal ligand-binding domain (LBD) of AR, encoded by exons 5-8, which initiates a cascade of events involving conformational changes and nuclear translocation, culminating in the binding of AR dimer to androgen response elements (AREs) located in the promoter and enhancer sequences of target genes. AR binds target AREs via its DNA-binding domain (DBD) which is composed of two zinc finger motifs, encoded by exons 2 and 3 , respectively. A portion of the DBD together with a downstream region in the hinge region, encoded by exon 4 , appears to confer nuclear localization signal (NLS). The LBD of most steroid hormone receptors contain Activation Function-2 (AF-2) that allows the recruitment of co-activators and co-repressors, resulting in the modulation of the transcriptional activities of receptors at target genes. The NH2-terminal domain (NTD), encoded by exon 1 , constitutes $\sim 60 \%$ of the $110-\mathrm{kDa}$ full-length protein and is the transcriptional regulatory region of the AR containing Activation Function-1 (AF-1). Unlike the other steroid hormone receptors, the transcriptional activity of the AR requires AF-1 in its NTD with negligible activity being attributed to AF-2 region in the LBD.

AR transactivation is a function of the interaction between the AR NTD and its $\mathrm{COOH}$ terminal domain (N-C interaction) following the binding of androgen to its LBD. AR is postulated to have a continued critical role in advanced prostate cancer. Mechanisms proposed for the continued transcriptional activity of the AR in spite of castrate levels of circulating testosterone include: gain-of-function mutations in the AR LBD that render antiandrogens agonistic, increased levels of AR or altered expression of co-regulators of AR within at least a subset of CRPC; ligand-independent activation of the AR through its NTD in the absence of ligand by alternative signaling pathways involving kinases and cytokines; residual androgens from various sources that include adrenal glands and the tumor itself (intratumoral); and perhaps most importantly expression of constitutively active splice variants of AR that lack the LBD. In the following sections, some of these mechanisms are described in the light of recent developments in a greater detail. AR pathobiology in prostate cancer has been extensively reviewed [4-7]. Here, recent developments in the field are reviewed.

\section{Efficacy versus resistance}

Historically, a lack of cure with traditional androgen ablation therapy has led some to question if the AR/androgen pathway is an ideal target for the treatment of advanced prostate cancer. Recent developments have strengthened the role of AR and provide evidence of poor efficacy and the inability of current drugs to inhibit all AR activity. While castration is effective in depleting gonadal androgens, tumor levels of androgen can remain relatively high; $\sim 25-50 \%$ by some estimates [8]. These elevated levels of intratumoral androgen appear in part to be due to intact adrenal and/or prostatic de novo biosynthesis of androgen [9-11], although the latter is not without controversy [12]. This suggests that inhibiting the synthesis of all androgen from both testicular and non-testicular sources might improve treatment of advanced prostate cancer. Indeed, the CYP17 inhibitor, Abiraterone, that blocks the synthesis of testicular and non-testicular androgens provides a 3.9 months survival advantage for the treatment of metastatic, CRPC in men who received prior 
docetaxel chemotherapy [13] and has been approved by the US Food and Drug Administration.

The combination of castration with anti-androgens is effective in the treatment of CRPC. However, currently approved anti-androgens bind the AR with relatively poor affinity compared to the physiological ligand DHT. Some antiandrogens such as bicalutamide can facilitate nuclear translocation of AR and binding to AREs. These features are particularly problematic given that AR is presumably hypersensitized in CRPC and also in the light of the higher intratumoral or adrenal androgens. Based on the scaffold of bicalutamide, an investigational anti-androgen MDV3100 has been developed which has improved affinity for AR and does not cause AR to translocate to the nucleus [14]. MDV3100 is effective in CRPC xenograft models that express full-length AR [14] and is now in Phase III clinical trials [15]. These clinical developments imply that more global and effective inhibitors of androgen biosynthesis and better affinity antiandrogens would significantly improve the management of advanced prostate cancer.

\section{Variants/isoforms of the androgen receptor in prostate cancer cells}

Early functional studies showed that deletion of the LBD results in constitutive activation of the AR [16]. Beyond structure-function analysis, the relevance of these findings are supported by the discovery of equivalent naturally occurring constitutively active splice variants of the AR that lack LBD in both clinical samples of prostate cancer and prostate cancer cell lines. Western blot analyses of protein lysates prepared from prostate cancer cell lines C-81/long passage LNCaP, CWR-R1, and 22RV1 cells using anti-AR antibodies to epitopes in the AR NTD detected lower molecular weight bands in the range of 70-90 kDa with weaker bands also detected in LNCaP and LAPC-4 cell-lines [17, 18]. Mapping using region-specific antibodies revealed that some of these AR isoforms lack the LBD $[17,18]$. This data served as a springboard for the identification of AR splice variants [19]. Since then, numerous transcript variants of AR from various prostate cancer lines, xenografts or clinical samples have been reported [2, 19-24]. Important features of such transcripts or cognate protein products are listed in Tables 1 and 2. Here, we focus on transcripts that have been detected in more than one prostate cancer specimens, cell lines or xenografts. These variants are depicted in Figure 2.

\section{Structure of variant transcripts and putative protein products}

All AR variants investigated thus far, with the exception of AR23 [20], lack the region encoding the LBD partially or entirely and the majority also lack the hinge region. Some variants such as $\mathrm{AR}^{\mathrm{Ex} 1 / 2 / 2 \mathrm{~b}}$ are also devoid of the second zing finger of the DBD [19]. ARV7/AR3 is the only variant to date that has been shown to encode for a detectable cognate protein endogenously using an antibody against a unique epitope (EKFRVGNCKHLKMTRP) that arises from cryptic exon 3 (also referred to as exon 3b) $[23,24]$. AR-V7/AR3 has intact NTD and DBD but lacks the hinge region and LBD. The majority of other variants also have predicted unique sequence and hence specific antibodies are in theory possible. These putative sequences are encoded by the novel exons corresponding to introns 2 and 3 of the full-length AR (fl-AR), or are due to frame shift arising from altered splicing. The multiplicity of protein bands detected at $\sim 80 \mathrm{kDa}$ using anti-AR antibodies targeted to the NTD, barring proteolysis or posttranslational modifications, suggest that other variants besides AR-V7/AR3 are also expressed. The presence of protein bands in some of the LuCaP panel of xenografts that are not recognized by anti-AR-V7 antibody [24] further supports this expectation. 


\section{Variant expression and associated growth advantages in CRPC-like conditions}

At the outset, it should be stated that the relative expression values between the different variants and fl-AR when analyzed by a PCR-approach may not be accurate due to unintended bias owing to differences in PCR primer efficiency and amplicon sizes. Some approaches such as absolute quantification by RT-PCR using in vitro transcribed template as standards [25] should reduce some of the technical bias. Comparison of variant levels with that of fl-AR also is complicated by the possibility of novel variants that are yet to be discovered, which may increase the proportion of aggregate levels of truncated variants lacking LBD. Indeed, a PCR-free approach that involved in vitro transcription coupled to a tiling microarray minimized, if not avoided, bias down-stream to Exon-3 to identify AR variants [2]. Since multiple probes spanning the AR locus were employed bias due to hybridization kinetics was minimal.

Variants are expressed in benign prostate epithelium, yet levels are increased in tumors and upon castration [22]. Full-length AR was detected in 35/36 samples of laser captured and microdissected $(\mathrm{LCM})$ benign prostate tissue from men $(\mathrm{n}=36)$ without prostate cancer, ages 35-55 years with serum levels of PSA less than $2 \mathrm{ng} / \mathrm{ml}$. A total of 10 samples (27.8\%) contained detectable levels of either V567es or V7. Importantly, expression of these variants in this cohort of men without prostate cancer was only detected in those whose levels of androgens were suppressed [22]. When comparing levels of expression of variants in primary (untreated) prostate cancer obtained by radical prostatectomy by LCM of matched benign and cancerous cells it was determined that: 1) variants are expressed in both the benign and malignant cells; and 2) variants are not necessarily etiologic in the initiation of prostate cancer. However, the level of expression of V7 in early-stage hormone naïve prostate cancer is associated with a worse prognosis after radical prostatectomy [24].

Examination of 69 metastases from 13 men who died of CRPC revealed that 23/69 samples were of neuroendocrine phenotype, which is negative for both fl-AR and variants [22]. Of the remaining 46 samples that expressed fl-AR or variant the following were observed: $80 \%$ (37/46) expressed prototype AR; and 73\% expressed V567es and/or V7. As much as 20\% of metastases expressed only V567es meaning that all current therapies that target the AR LBD would have no effect on these metastases. Twelve of 13 patients had a minimum of 1 metastasis that was positive for at least 1 variant while 10/13 had at least a minimum of one metastasis that was positive for V567es. No patient had all samples positive for the same variant. A more recent study has demonstrated the expression of V1, V7 and V567es in bone metastasis specimens [26]. Importantly, this study also shows that the expression of each of the three variants is enriched in CRPC bone metastasis samples versus those isolated from bone lesions of hormone-naïve patients.

Over expression of $\mathbf{V 7}$ in LNCaP cells and specific depletion of endogenous variants in 22Rv1 cells results in increased and decreased growth, respectively, under CRPC-like in vitro and in vivo conditions [19,21,23]. Interestingly, V7 knockdown reduced proliferation but had little effects on apoptosis [23]. V567es increases proliferation of LNCaP cells in the absence of androgen as well as enhanced proliferation in response to very low levels of androgen [22]. In intact, non-castrated animals there were no differences in tumor growth between LNCaP and LNCaP that stably expressed V567es. However, in castrated hosts, V567es tumours were larger than tumours without V567es. This implies a growth advantage under castrate conditions when the variant is expressed. Consistent with this interpretation, levels of expression of variant correlated with growth in castrated mice among LuCaP xenograft models [22]. Thus, the expression of these variants is predicted to be a mechanism of resistance to current hormonal therapies and development of CRPC. In support of this 
prediction, V7 is expressed at higher levels in CRPC [23, 24] as are V1, V9, and V12 [2, 24]. Noteworthy, V12 has identical coding sequence with V567es [2]. Specifically, the expression values for fl-AR, V1, V7, V9, V12 were elevated in CRPC by 11-, 22-, 20-, 3-, and $\sim 4$-fold, respectively, when compared with hormone-naive prostate cancer. No variant protein was detected in VCaP tumours using an antibody to the AR NTD in intact mice. However, both an increase in fl-AR and $\mathbf{V 7}$ occurred by 2 days post-castration reaching maximum levels at 14 days, which was ablated by testosterone replacement. Thus, the androgen environment plays a key role in the regulating the expression of variants, which in turn provides a growth advantage under castrate conditions.

\section{Interaction with full-length AR}

All the nuclear variants tested so far have transcriptional activity in-and-by themselves. However, variants with truncated LBD can be co-expressed with fl-AR in various cell lines, xenografts and clinical samples. Thus, the question whether the variants interact with fl-AR is highly relevant. V567es is constitutively active and nuclear [22]. V567es and fl-AR do interact when cotransfected into the AR negative M12 prostate cancer cell line [22]. Perhaps due to this interaction with fl-AR, expression of V567es results in an increased stability of fl-AR protein as well as causes nuclear localization of fl-AR in the absence of androgens [22]. However, V7 has been shown not to interact with fl-AR when examining endogenous proteins in 22Rv1 cells [23]. Differences in interaction with fl-AR between V567es and V7 might be due to V7 lacking exon 4 that encodes the hinge region that is present in AR567es and/or the unique C-terminus short peptide sequence in V7 although cell specificity cannot be ruled out. The third zinc finger motif present in the fl-AR in 22Rv1 [18], which has not been detected in any other clinical or post-clinical material as of yet, might also present a confounding factor by impeding interactions that could potentially be different than that observed with the wild-type fl-AR. In vitro, $\mathbf{V 7}$ overexpression in LNCaP that contains flAR confers anchorage-independent growth in the absence of androgens [21]. Remarkably, this phenotype was reversed upon treatment with the LBD-dependent AR inhibitor MDV3100 implicating a requirement for fl-AR [21], despite the observation that V7 apparently does not interact with fl-AR. If $\mathrm{V} 7$ requires fl-AR for activity, one would predict that depleting fl-AR using siRNA specific to AR LBD in a cell line that expresses functional variant(s) lacking LBD would lead to abrogation of constitutive activity associated with variant expression. Contrary to this prediction, fl-AR is not required for variant transcriptional activity as shown by application of LBD-specific siRNA in LNCaP cells that ectopically expressed V7 [2]. Consistent with the conclusion that at least V7 does not require fl-AR, variant-associated growth of 22Rv1 cells under CRPC conditions is not dependent on fl-AR as shown by knocking down its expression using two independent siRNAs targeting exons encoding AR LBD [27]. Importantly, in both studies, androgendependent activity was blocked upon transfection of the siRNAs specific to exons encoding AR LBD. Collectively, these observations from two independent model systems together with variant expression in CRPC samples in the absence of fl-AR strongly suggest that the activities of at least some variants do not require expression of fl-AR.

\section{Sub-cellular localization}

Structurally similar AR variants may have distinct biological activity thereby necessitating research into understanding the biology of each variant and how these variants interact with one-another. One obvious difference is that the different variants can be localized in different cellular compartments which may be cell-specific or depend on the presence of flAR. V7 provides an interesting example of a variant that has conditional cellular localization [23]. V7 can be nuclear in cultured prostate cancer cells under androgen-depleted conditions and constitutively active in driving the expression of genes including canonical androgen- 
responsive genes using reporter assays and microarray analysis. However, examination of clinical tissues has revealed a more complicated pattern of expression. In benign prostate tissues, V7 mainly stained basal and stromal cells while the luminal cells were barely stained [23]. In malignant glands, $\mathbf{V 7}$ stained the majority of luminal cells in the cytoplasm and was nuclear in CRPC tissues [23]. Cytoplasmic expression of $\mathbf{V 7}$ was in $86 \%$ of cases which suggests additional variables are required for its nuclear localization [23].

V1 is cytoplasmic regardless of androgen status when ectopically expressed in Cos-7 cells [21]. V9 is also exclusively cytoplasmic [2]. V12 and V567es are unique in that they retain the hinge region necessary for nuclear translocation [2, 22]. As expected, V12 and V567es localized to the nucleus regardless of presence of ligand $[2,22]$ in a manner similar to that first reported for the deletion construct AR1-653 engineered to map AR structure with activity [16]. The fl-AR translocates to the nucleus with V567es regardless of ligand [22]. All of the nuclear variants described so far display transcriptional activity as assayed with reporters or endogenous genes that contain AREs in their regulatory regions.

\section{Dynamics of AR target genes}

The correlation of androgen-regulated genes with CRPC gene expression signature has been a challenge. One potential explanation for this comes from the recent discovery that AR target genes in CRPC conditions are distinct from those that are induced by androgens in androgen-sensitive prostate cancer cell lines [28]. A compelling mechanism for such dynamics of AR-mediated gene expression is that AR differentially associates with various transcription factors and co-regulators [28, 29]. These findings, even though they remain to be corroborated with independent model systems, have further strengthened the postulate that AR has a critical role in CRPC.

Cell-specific and gene-specific gene expression is thought to result from recruitment of different co-regulatory proteins to the AR. Therefore it would be expected that variants lacking specific regions of the AR would be devoid of protein interfaces and/or have new interfaces for the recruitment of different subsets of interacting proteins and possibly posttranslational modifications. Expression of V567es increases levels of endogenous genes both in the absence and presence of DHT in LNCaP cells co-expressing both variant and fl-AR. These genes included well-known androgen regulated genes such as PSA, TMPRSS2, FKBP5, and NKX3.1 [22]. Importantly, STAT3 and JUN were increased by V567es [22]. As predicted, V567es heterodimer with fl-AR had a different gene expression profile than solely fl-AR in response to DHT. Some differences could be attributed to V567es abrogating non-genomic signalling in response to androgen by a mechanism that diminishes cytoplasmic levels of fl-AR. IGF-IR is enhanced by non-genomic signalling through androgen stimulation of fl-AR binding Src and downstream activation of MAPK. V567es did not increase IGF-IR. V7 also regulates a unique set of genes such as AKT1, which is not regulated by fl-AR, while still increasing PSA mRNA in LNCaP cells which could not be blocked by the antiandrogen bicalutamide [23]. Mechanistic differences were observed using chromatin immunoprecipitation assays. V7 physically interacted with an ARE in the AKT1 locus while the fl-AR does not bind to this ARE [23]. Curiously, when examining the well-characterized AREs on the PSA locus, V7 failed to bind to enhancer ARE but did bind the promoter ARE in both CWR-R1 and 22RV1 [23]. Knockdown of V7 altered expression of a set of 188 genes in 22RV1 and CWR-R1 prostate cancer cells. Of these, 71 genes were commonly regulated by both V7 and fl-AR (e.g., IGFBP3, FKBP5). The AR target genes CLU, TMEPAI, PSA and CLDN4 were not affected by knockdown of V7. Interestingly, V7 regulates expression of MAP4K4, HOXB7, and ELK1 that are increased in CRPC or metastases. In LNCaP cells, V7 induced canonical androgen-responsive genes, such as PSA, KLK2, NKX3-1, FKBP5, and TMPRSS2, in the absence of androgens. Thus, differences in 
gene expression profiles are governed by variants versus fl-AR with some overlap. This mirrors and may even partly explain the subset of androgen-regulated genes in CRPC versus androgen-dependent models. Clinically, these differences in gene expression profiles may reveal biomarkers to identify patents with tumors that express variants and would not respond to therapies that target the AR LBD.

\section{Regulation of variant isoform production}

The first mechanism forwarded for the production of the naturally occurring AR isoforms lacking LBD in CWR22 cell lines was calpain-mediated proteolysis [30, 31] although not without contradictory data [32]. However, it is now evident that splicing events generates some variants. Given the androgen regulation of expression of variant, alternative splicing allowing exon-skipping, intron retention or both might be at play. If so, elucidating these mechanisms can potentially identify possibilities of intercepting the production of these presumably deadly forms of AR. An alternative mechanism for the generation of AR isoforms lacking LBD involves intragenic rearrangement within the AR locus within a region that is downstream to exon 2 and upstream to exon 4 in $22 \mathrm{Rv} 1$ cells with data indicative of related events in clinical CRPC samples [25]. Interestingly, in CWR22Pc, an androgen-dependent form of CWR22, the intragenic rearrangement does not appear to be present and this cell-line does not express the truncated variants at a significant level. Remarkably, within days of culture under androgen-free conditions, CWR22pc displayed readily detectable level of the truncated variants. This expression of variant appears to be preceded by the aforementioned intragenic rearrangement. The association of a genetic event with levels of the AR variants beyond the CWR22 model system is required to determine the clinical importance of this mechanism. In the meantime, an attractive hypothesis has been suggested where the genetic rearrangement events render exon 4 and downstream regions more drastically distal to the transcription site; which somehow alters the splicing dynamics causing it to halt earlier [25]. While this mechanism remains a possibility for a number of the variants, it is unclear how it would explain expression of ARV12, AR-V13, AR-V14, and v567es which display deviant events downstream of exon 4. Nevertheless, cognizant of the proposed mechanism and the hormonal regulation of the variants, it is tempting to seek an analogy with the recently identified mechanism of ARinducible TMPRSS2: ERG fusion generation [33-35]. Could castration-induced transcriptional events set the stage for the rearrangement thereby leading to aberrant splicing?

\section{Complexity or noise?}

An alternative explanation for the presence of AR variant transcripts is that they are stochastic byproducts of transcriptional and/or splicing noise at the AR locus. Cryptic products can be degraded by non-sense mediated decay and other nuclear quality control mechanisms. The presence of variant mRNAs may be an indication of escape from quality control mechanisms and perhaps implicate some biological pressure to maintain their expression. In the case of $\mathrm{V} 7$, a protein product has been detected and thus the generation of this variant is less likely to be non-deterministic. The functions assigned to particular variants and their differential expression during disease progression is further testimony to the pathophysiolocal relevance of the expression of at least some variants. However, paradoxical aspects of some of the variants do exist and are worth considering.

V1 is cytoplasmic and has a dominant negative function when co-expressed with V7 [21], yet V1 is detectable in clinical samples with as strong differential expression in CPRC as flAR or V7 [24] [26]. V1 and V9 do not have transcriptional activity when expressed in PC-3 cells, nor does V1 have transcriptional activity in DU145 cells [21]. In contrast, in LNCaP 
cells both V1 and V9 have constitutive transcriptional activity, apparently independent of flAR, emphasizing cell-specific differences in activity.

AR23 possesses a splicing-related insertion that disrupts its DBD but otherwise is identical to fl-AR [20,36]. Despite the predicted lack of DNA binding function [37], expression of AR23 in LNCaP cells increased transcriptional activity at AR promoters and at promoters that are regulated by other transcription factors including NF-kB [20,36].

$\mathbf{A} \mathbf{R}^{\mathbf{E x} 1 / 2 \mathbf{b}}$, predicted to lack DBD and LBD, is detectable at the transcript level in two different CRPC forms of the LuCaP panel of xenografts and in the LAPC4 cell line [19]. The prevalence of $\mathbf{A R}^{\mathbf{E x} \mathbf{1} / \mathbf{2 b}}$ transcripts may be underestimated because the approaches to discover AR variants screen for Exon 2 and downstream regions.

Inactive forms of AR that lack part of the AR LBD, V13 and V14 have also been detected in CRPC samples [20, 36]. Based on previous analysis of Cis-determinants of AR subcellular localization [38], these variants are unlikely to have nuclear localization.

Expression of variants that are dysfunctional or potentially inhibitory to the function of flAR may serve to provide a negative feedback mechanism. Alternatively, the relative levels of variant to fl-AR may yield differential responses, or activity may be cell-specific. Very low levels of variants may interact with fl-AR to potentiate AR signaling while high levels of variant relative to fl-AR may repress.

A more general question that arises is what is the need for prostate cancer to express AR variants with truncated LBD? One explanation is that the presence of LBD in unliganded $\mathrm{AR}$, when androgens are in a limiting concentration as in CRPC, inhibits nuclear translocation or facilitates cytoplasmic sequestration and/or inhibits NTD activity. However, this is at most merely part of the explanation for the following reasons: (1) disparate gene expression signature between the variants and fl-AR (2) presence of the variants in other phases of the disease and (3) variants such as V1 and V9 being cytosolic. Interestingly, Kaku and co-workers demonstrated that the NTD and LBD, in the absence of DBD and hinge region, co-operate to facilitate cytoplasmic retention [39]. Previous studies have shown that the LBD contains nuclear export signal, which might in part explain why unliganded fl-AR tends to be cytoplasmic [38, 40]. Intriguingly, the NTD also harbors a nuclear localization signal, albeit not as strong as those located in the region spanning DBD and hinge region [39]. Thus, AR variants devoid of LBD should yield AR species unable to be exported to the cytoplasm with the NTD NLS(s) and hinge region potentially able to confer constitutive nuclear localization in the absence of androgen. If so, the most parsimonious explanation for cell-specific cytoplasmic localization of V1 and V9 might be inhibition of NTD NLS or independent cytoplasmic retention capacity due to their unique Cterminal extension.

\section{AR NTD and implications for CRPC treatment}

AR NTD accounts for majority of AR transcriptional activity in the presence or absence of androgens. Targeting the AR NTD with decoy molecules has shown antitumor activity in vivo [41]. Current hormonal therapies all target the AR LBD either by reducing ligand with castration and CYP17 inhibitors or application of antiandrogens. These approaches all eventually fail by mechanisms that may involve the expression of constitutively active splice AR variants that lack the LBD. AR is activated via its NTD by alternative signaling mechanisms including the IL- 6 and PKA pathways in the absence of androgens and serum; thus in conditions free of any of the precursors for androgen biosynthesis [42-44]. Castration-induced expression of some variants is inversely correlated to the residual levels of intratumoral androgen [22]. The lower the residual levels of androgens, the higher the 
expression of the splice variants. Expression of constitutively active AR variant may be a compensatory mechanism for low castrate levels of androgen. Thus there is a strong rationale for developing small molecules that specifically bind the AR NTD to block activity.

One challenge in developing antagonists of the AR NTD has been the fact that this region of the AR is intrinsically disordered and thus not amenable to crystallographic structural determinations, which in turn precludes virtual screening of small molecule libraries. Recent studies have circumvented this problem by using cell-based screening protocols [45, 46]. One NTD antagonist, EPI-001, is well-characterized in pre-clinical studies [45, 47]. Treatment of CRPC xenograft tumors with EPI-001 caused tumor regression without any apparent general toxicity [45]. Antiandrogens do not cause significant apoptosis that may forecast their potential failure in the clinic. In contrast, EPI-001 causes tumor regression by inducing apoptosis and reducing proliferation that may be extrapolated to be indicative of better tumor control in the clinic. Results to date for EPI-001 provide a strong proof-ofprinciple that NTD inhibitors can indeed be developed and display strong activity against prostate cancer.

\section{Conclusion}

Tremendous advances have been achieved in both the quantity and insightful quality of evidence for the potential of exploiting the dependency of prostate cancer on all AR species. Significant paradigm shift has occurred towards achieving an "androgen-free" CRPC as well as in exploiting the requirement of the NTD for AR activity with evidence that this domain is a "druggable" target. Together with the discovery of antiandrogens with several orders of magnitude better affinity compared to previous anti-androgens, these advancements represent a striking step forward in the development of therapies for advanced prostate cancer. There is now compelling evidence that constitutively active AR variants are yet another aspect of AR-related mechanism underlying CRPC, although much work is still required to determine the function and mechanisms of the individual variants. As the outcome of the ongoing and future clinical trials of the next-generation AR targeting drugs is assessed, a due consideration should be given to these variants and thereby determine the extent to which their expression in patients accounts for not only to castration-resistant but also androgen-independence mechanisms. These data may aid to stratify patients that would require treatment with AR NTD inhibitors subsequent, or simultaneous, with approaches that target the LBD or androgen biosynthesis.

\section{Acknowledgments}

This work was supported by grants from the Canadian Institutes of Health Research (MOP-84325) and the US National Cancer Institute (2R01 CA105304).

\section{References}

1. Chen CD, Welsbie DS, Tran C, Baek SH, Chen R, Vessella R, Rosenfeld MG, Sawyers CL. Molecular determinants of resistance to antiandrogen therapy. Nat Med. 2004; 10(1):33-39. [PubMed: 14702632]

2. Hu R, Isaacs WB, Luo J. A snapshot of the expression signature of androgen receptor splicing variants and their distinctive transcriptional activities. The Prostate. 201110.1002/pros.21382

3. Ostling P, Leivonen SK, Aakula A, Kohonen P, Makela R, Hagman Z, Edsjo A, Kangaspeska S, Edgren H, Nicorici D, et al. Systematic Analysis of MicroRNAs Targeting the Androgen Receptor in Prostate Cancer Cells. Cancer research. 2011; 71(5):1956-1967. [PubMed: 21343391]

4. Knudsen KE, Scher HI. Starving the addiction: new opportunities for durable suppression of AR signaling in prostate cancer. Clin Cancer Res. 2009; 15(15):4792-4798. [PubMed: 19638458] 
5. Dehm SM, Tindall DJ. Androgen receptor structural and functional elements: role and regulation in prostate cancer. Molecular endocrinology. 2007; 21(12):2855-2863. [PubMed: 17636035]

6. Wang G, Sadar MD. Amino-terminus domain of the androgen receptor as a molecular target to prevent the hormonal progression of prostate cancer. J Cell Biochem. 2006; 98(1):36-53. [PubMed: 16440300]

7. Niu Y, Chang TM, Yeh S, Ma WL, Wang YZ, Chang C. Differential androgen receptor signals in different cells explain why androgen-deprivation therapy of prostate cancer fails. Oncogene. 2010; 29(25):3593-3604. [PubMed: 20440270]

8. Labrie F, Cusan L, Gomez JL, Martel C, Berube R, Belanger P, Belanger A, Vandenput L, Mellstrom D, Ohlsson C. Comparable amounts of sex steroids are made outside the gonads in men and women: strong lesson for hormone therapy of prostate and breast cancer. The Journal of steroid biochemistry and molecular biology. 2009; 113(1-2):52-56. [PubMed: 19073258]

9. Locke JA, Guns ES, Lubik AA, Adomat HH, Hendy SC, Wood CA, Ettinger SL, Gleave ME, Nelson CC. Androgen levels increase by intratumoral de novo steroidogenesis during progression of castration-resistant prostate cancer. Cancer research. 2008; 68(15):6407-6415. [PubMed: 18676866]

10. Montgomery RB, Mostaghel EA, Vessella R, Hess DL, Kalhorn TF, Higano CS, True LD, Nelson PS. Maintenance of intratumoral androgens in metastatic prostate cancer: a mechanism for castration-resistant tumor growth. Cancer research. 2008; 68(11):4447-4454. [PubMed: 18519708]

11. Stanbrough M, Bubley GJ, Ross K, Golub TR, Rubin MA, Penning TM, Febbo PG, Balk SP. Increased expression of genes converting adrenal androgens to testosterone in androgenindependent prostate cancer. Cancer research. 2006; 66(5):2815-2825. [PubMed: 16510604]

12. Hofland J, van Weerden WM, Dits NF, Steenbergen J, van Leenders GJ, Jenster G, Schroder FH, de Jong FH. Evidence of limited contributions for intratumoral steroidogenesis in prostate cancer. Cancer research. 2010; 70(3):1256-1264. [PubMed: 20086173]

13. Attard G, Richards J, de Bono JS. New strategies in metastatic prostate cancer: targeting the androgen receptor signaling pathway. Clin Cancer Res. 2011; 17(7):1649-1657. [PubMed: 21372223]

14. Tran C, Ouk S, Clegg NJ, Chen Y, Watson PA, Arora V, Wongvipat J, Smith-Jones PM, Yoo D, Kwon A, et al. Development of a second-generation antiandrogen for treatment of advanced prostate cancer. Science. 2009; 324(5928):787-790. [PubMed: 19359544]

15. Scher HI, Beer TM, Higano CS, Anand A, Taplin ME, Efstathiou E, Rathkopf D, Shelkey J, Yu EY, Alumkal J, et al. Antitumour activity of MDV3100 in castration-resistant prostate cancer: a phase 1-2 study. Lancet. 2010; 375(9724):1437-1446. [PubMed: 20398925]

16. Jenster G, van der Korput HA, van Vroonhoven C, van der Kwast TH, Trapman J, Brinkmann AO. Domains of the human androgen receptor involved in steroid binding, transcriptional activation, and subcellular localization. Molecular endocrinology. 1991; 5(10):1396-1404. [PubMed: 1775129]

17. Gregory CW, He B, Wilson EM. The putative androgen receptor-A form results from in vitro proteolysis. Journal of molecular endocrinology. 2001; 27(3):309-319. [PubMed: 11719283]

18. Tepper CG, Boucher DL, Ryan PE, Ma AH, Xia L, Lee LF, Pretlow TG, Kung HJ. Characterization of a novel androgen receptor mutation in a relapsed CWR22 prostate cancer xenograft and cell line. Cancer research. 2002; 62(22):6606-6614. [PubMed: 12438256]

19. Dehm SM, Schmidt LJ, Heemers HV, Vessella RL, Tindall DJ. Splicing of a novel androgen receptor exon generates a constitutively active androgen receptor that mediates prostate cancer therapy resistance. Cancer research. 2008; 68(13):5469-5477. [PubMed: 18593950]

20. Jagla M, Feve M, Kessler P, Lapouge G, Erdmann E, Serra S, Bergerat JP, Ceraline J. A splicing variant of the androgen receptor detected in a metastatic prostate cancer exhibits exclusively cytoplasmic actions. Endocrinology. 2007; 148(9):4334-4343. [PubMed: 17540719]

21. Watson PA, Chen YF, Balbas MD, Wongvipat J, Socci ND, Viale A, Kim K, Sawyers CL. Constitutively active androgen receptor splice variants expressed in castration-resistant prostate cancer require full-length androgen receptor. Proceedings of the National Academy of Sciences of the United States of America. 2010; 107(39):16759-16765. [PubMed: 20823238] 
22. Sun S, Sprenger CC, Vessella RL, Haugk K, Soriano K, Mostaghel EA, Page ST, Coleman IM, Nguyen HM, Sun H, et al. Castration resistance in human prostate cancer is conferred by a frequently occurring androgen receptor splice variant. The Journal of clinical investigation. 2010; 120(8):2715-2730. [PubMed: 20644256]

23. Guo Z, Yang X, Sun F, Jiang R, Linn DE, Chen H, Chen H, Kong X, Melamed J, Tepper CG, et al. A novel androgen receptor splice variant is up-regulated during prostate cancer progression and promotes androgen depletion-resistant growth. Cancer research. 2009; 69(6):2305-2313. [PubMed: 19244107]

24. Hu R, Dunn TA, Wei S, Isharwal S, Veltri RW, Humphreys E, Han M, Partin AW, Vessella RL, Isaacs WB, et al. Ligand-independent androgen receptor variants derived from splicing of cryptic exons signify hormone-refractory prostate cancer. Cancer research. 2009; 69(1):16-22. [PubMed: 19117982]

25. Li Y, Alsagabi M, Fan D, Bova GS, Tewfik AH, Dehm SM. Intragenic Rearrangement and Altered RNA Splicing of the Androgen Receptor in a Cell-Based Model of Prostate Cancer Progression. Cancer research. 2011; 71(6):2108-17. [PubMed: 21248069]

26. Hornberg E, Ylitalo EB, Crnalic S, Antti H, Stattin P, Widmark A, Bergh A, Wikstrom P. Expression of Androgen Receptor Splice Variants in Prostate Cancer Bone Metastases is Associated with Castration-Resistance and Short Survival. PLoS One. 2011; 6(4):e19059. [PubMed: 21552559]

27. Mashima T, Okabe S, Seimiya H. Pharmacological targeting of constitutively active truncated androgen receptor by nigericin and suppression of hormone-refractory prostate cancer cell growth. Molecular pharmacology. 2010; 78(5):846-854. [PubMed: 20709811]

28. Wang Q, Li W, Zhang Y, Yuan X, Xu K, Yu J, Chen Z, Beroukhim R, Wang H, Lupien M, et al. Androgen receptor regulates a distinct transcription program in androgen-independent prostate cancer. Cell. 2009; 138(2):245-256. [PubMed: 19632176]

29. Heemers HV, Schmidt LJ, Sun Z, Regan KM, Anderson SK, Duncan K, Wang D, Liu S, Ballman $\mathrm{KV}$, Tindall DJ. Identification of a clinically relevant androgen-dependent gene signature in prostate cancer. Cancer research. 2011; 71(5):1978-1988. [PubMed: 21324924]

30. Libertini SJ, Tepper CG, Rodriguez V, Asmuth DM, Kung HJ, Mudryj M. Evidence for calpainmediated androgen receptor cleavage as a mechanism for androgen independence. Cancer research. 2007; 67(19):9001-9005. [PubMed: 17909000]

31. Chen H, Libertini SJ, Wang Y, Kung HJ, Ghosh P, Mudryj M. ERK regulates calpain 2-induced androgen receptor proteolysis in CWR22 relapsed prostate tumor cell lines. The Journal of biological chemistry. 2010; 285(4):2368-2374. [PubMed: 19946123]

32. Sivanandam A, Murthy S, Chinnakannu K, Bai U, Kim SH, Barrack ER, Menon M, Reddy GP. Calmodulin protects androgen receptor from calpain-mediated breakdown in prostate cancer cells. Journal of cellular physiology. 2011; 226(7):1889-96. [PubMed: 21506119]

33. Bastus NC, Boyd LK, Mao X, Stankiewicz E, Kudahetti SC, Oliver RT, Berney DM, Lu YJ. Androgen-induced TMPRSS2:ERG fusion in nonmalignant prostate epithelial cells. Cancer research. 2010; 70(23):9544-9548. [PubMed: 20947519]

34. Lin C, Yang L, Tanasa B, Hutt K, Ju BG, Ohgi K, Zhang J, Rose DW, Fu XD, Glass CK, et al. Nuclear receptor-induced chromosomal proximity and DNA breaks underlie specific translocations in cancer. Cell. 2009; 139(6):1069-1083. [PubMed: 19962179]

35. Mani RS, Tomlins SA, Callahan K, Ghosh A, Nyati MK, Varambally S, Palanisamy N, Chinnaiyan AM. Induced chromosomal proximity and gene fusions in prostate cancer. Science. 2009; 326(5957):1230. [PubMed: 19933109]

36. Steinkamp MP, O’Mahony OA, Brogley M, Rehman H, Lapensee EW, Dhanasekaran S, Hofer MD, Kuefer R, Chinnaiyan A, Rubin MA, et al. Treatment-dependent androgen receptor mutations in prostate cancer exploit multiple mechanisms to evade therapy. Cancer research. 2009; 69(10): 4434-4442. [PubMed: 19366804]

37. Wickert L, Selbig J. Structural analysis of the DNA-binding domain of alternatively spliced steroid receptors. J Endocrinol. 2002; 173(3):429-436. [PubMed: 12065232]

38. Zhou ZX, Sar M, Simental JA, Lane MV, Wilson EM. A ligand-dependent bipartite nuclear targeting signal in the human androgen receptor. Requirement for the DNA-binding domain and 
modulation by NH2-terminal and carboxyl-terminal sequences. The Journal of biological chemistry. 1994; 269(18):13115-13123. [PubMed: 8175737]

39. Kaku N, Matsuda K, Tsujimura A, Kawata M. Characterization of nuclear import of the domainspecific androgen receptor in association with the importin alpha/beta and Ran-guanosine $5^{\prime}$ triphosphate systems. Endocrinology. 2008; 149(8):3960-3969. [PubMed: 18420738]

40. Saporita AJ, Zhang Q, Navai N, Dincer Z, Hahn J, Cai X, Wang Z. Identification and characterization of a ligand-regulated nuclear export signal in androgen receptor. The Journal of biological chemistry. 2003; 278(43):41998-42005. [PubMed: 12923188]

41. Quayle SN, Mawji NR, Wang J, Sadar MD. Androgen receptor decoy molecules block the growth of prostate cancer. Proceedings of the National Academy of Sciences of the United States of America. 2007; 104(4):1331-1336. [PubMed: 17227854]

42. Ueda T, Mawji NR, Bruchovsky N, Sadar MD. Ligand-independent activation of the androgen receptor by interleukin- 6 and the role of steroid receptor coactivator-1 in prostate cancer cells. The Journal of biological chemistry. 2002; 277(41):38087-38094. [PubMed: 12163482]

43. Ueda T, Bruchovsky N, Sadar MD. Activation of the androgen receptor N-terminal domain by interleukin-6 via MAPK and STAT3 signal transduction pathways. The Journal of biological chemistry. 2002; 277(9):7076-7085. [PubMed: 11751884]

44. Sadar MD. Androgen-independent induction of prostate-specific antigen gene expression via crosstalk between the androgen receptor and protein kinase A signal transduction pathways. The Journal of biological chemistry. 1999; 274(12):7777-7783. [PubMed: 10075669]

45. Andersen RJ, Mawji NR, Wang J, Wang G, Haile S, Myung JK, Watt K, Tam T, Yang YC, Banuelos CA, et al. Regression of castrate-recurrent prostate cancer by a small-molecule inhibitor of the amino-terminus domain of the androgen receptor. Cancer Cell. 2010; 17(6):535-546. [PubMed: 20541699]

46. Sadar MD, Williams DE, Mawji NR, Patrick BO, Wikanta T, Chasanah E, Irianto HE, Soest RV, Andersen RJ. Sintokamides A to E, chlorinated peptides from the sponge Dysidea sp. that inhibit transactivation of the N-terminus of the androgen receptor in prostate cancer cells. Organic letters. 2008; 10(21):4947-4950. [PubMed: 18834139]

47. Sadar MD. Small molecule inhibitors targeting the "Achilles' Heel” of androgen receptor activity. Cancer research. 2011; 71(4):1208-1213. [PubMed: 21285252] 


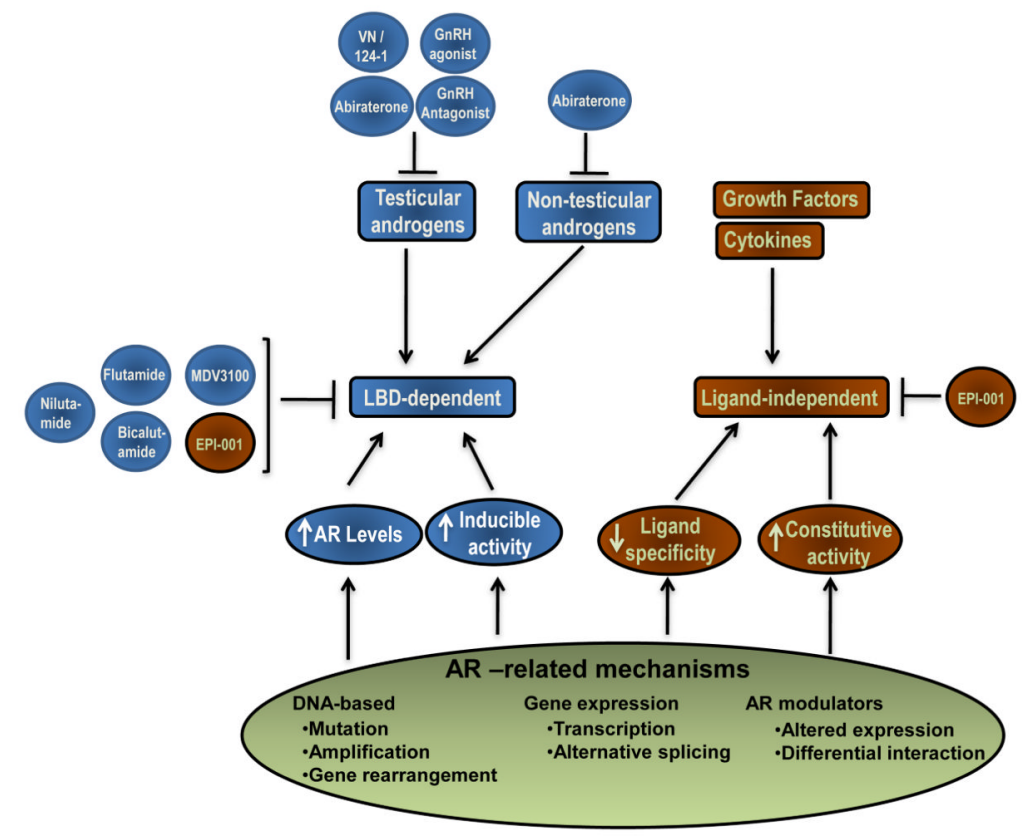

Figure 1. Drug targets of AR-mediated mechanisms underlying CPRC

Drugs known to directly bind AR or block enzymes catalyzing the synthesis of androgen are shown. EPI-001 is predicted to intercept with all facets of AR associated with CPRC due to the requirement of the NTD for AR transcriptional activity. 


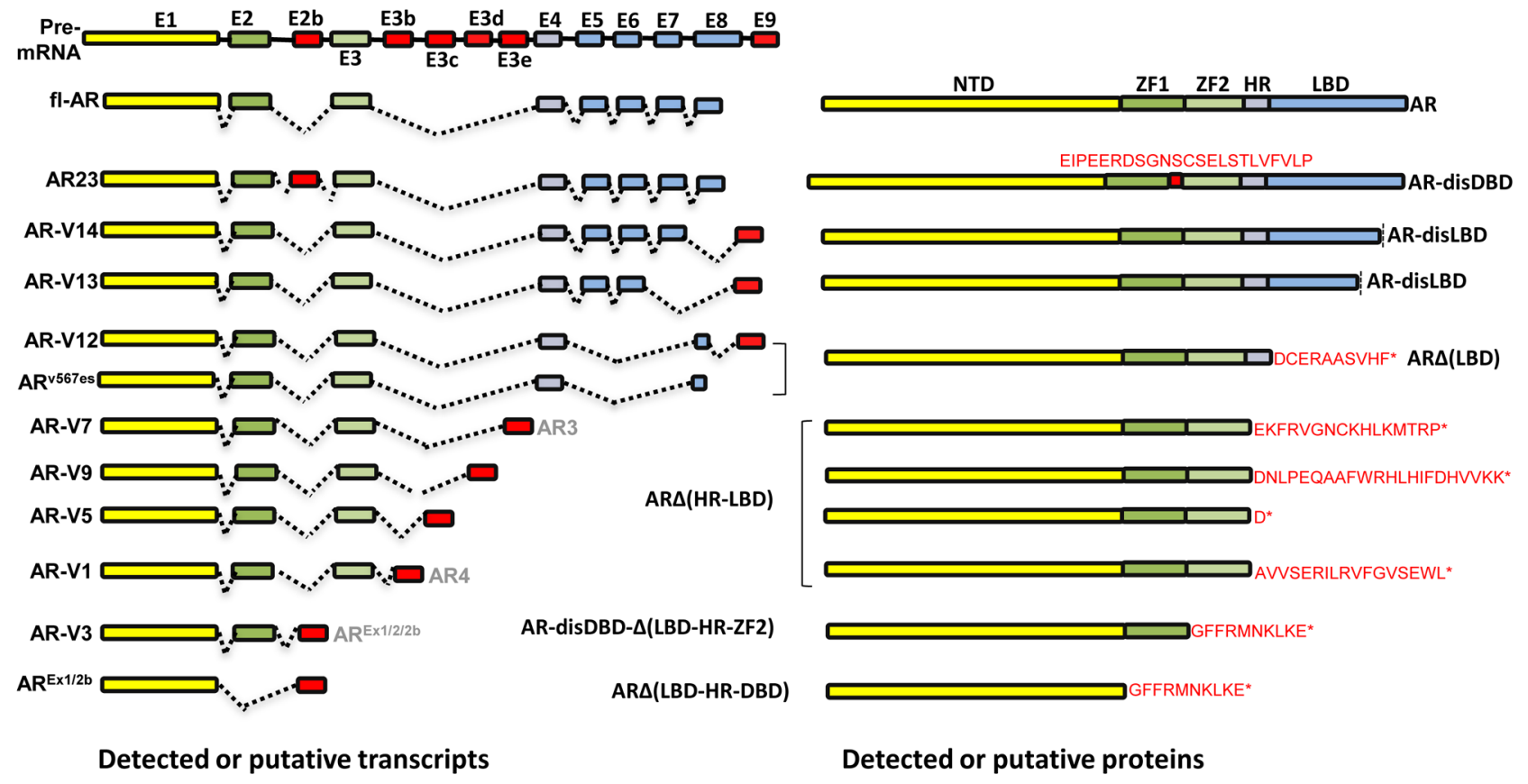

Figure 2. Variant transcripts and predicted or detected cognate protein products Despite uniform color code (red), the resulting predicted/detected peptide sequences are unique to each variant. Alternative names are indicated in gray font. Nomenclature of novel exons is based on the relative position in the AR locus. "dis" designates disrupted domain. Novel exons and related peptide sequences are indicated in red. Depictions are not to scale. Those variants that are reportedly detected in only one prostate cancer specimen or derivatives thereof are not shown. 


\begin{tabular}{|c|c|c|c|c|c|c|c|c|c|c|c|c|c|c|c|c|c|}
\hline 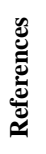 & 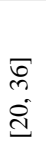 & $\begin{array}{l}\vec{J} \\
\mathcal{d} \\
\mathcal{d}\end{array}$ & $\stackrel{F}{I}$ & $\mid \begin{array}{l}\bar{J} \\
\stackrel{\Xi}{\Xi}\end{array}$ & $\begin{array}{l}\text { F } \\
\text { J } \\
\text { ปे } \\
\stackrel{\Xi}{\Xi}\end{array}$ & I & $\underset{\Xi}{\mathbb{I}}$ & $\begin{array}{l}\vec{J} \\
\tilde{d} \\
\vec{d}\end{array}$ & $\bar{\Xi}$ & $\begin{array}{l}\bar{\sim} \\
\dot{d}\end{array}$ & $\bar{\Xi}$ & $\bar{\Xi}$ & $\bar{\Xi}$ & $\Xi$ & $\bar{\Xi}$ & $\overline{\mathrm{d}}$ & $\bar{\Xi}$ \\
\hline 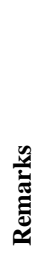 & & & 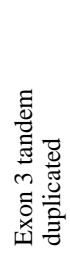 & 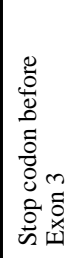 & 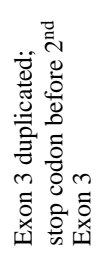 & & & 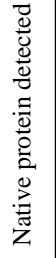 & & & & & & & & 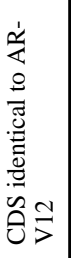 & \\
\hline 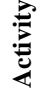 & 8 & Өి & & 0 & u & & & u & & 8 & & & 0 & - & & $u$ & \\
\hline 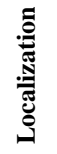 & $\vec{u}$ & $\vec{U}$ & & & & & & $\underset{*}{\grave{z}}$ & & $\vec{U}$ & & & $\mathrm{z}$ & & & z & \\
\hline $\bar{E}$ & 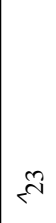 & $\stackrel{2}{2}$ & 2 & $n$ & $\tilde{n}$ & - & 6 & 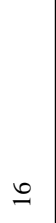 & 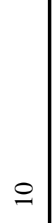 & $\ddot{\lambda}$ & ले & ¿i & 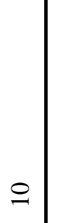 & $m$ & -1 & 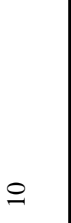 & \\
\hline
\end{tabular}

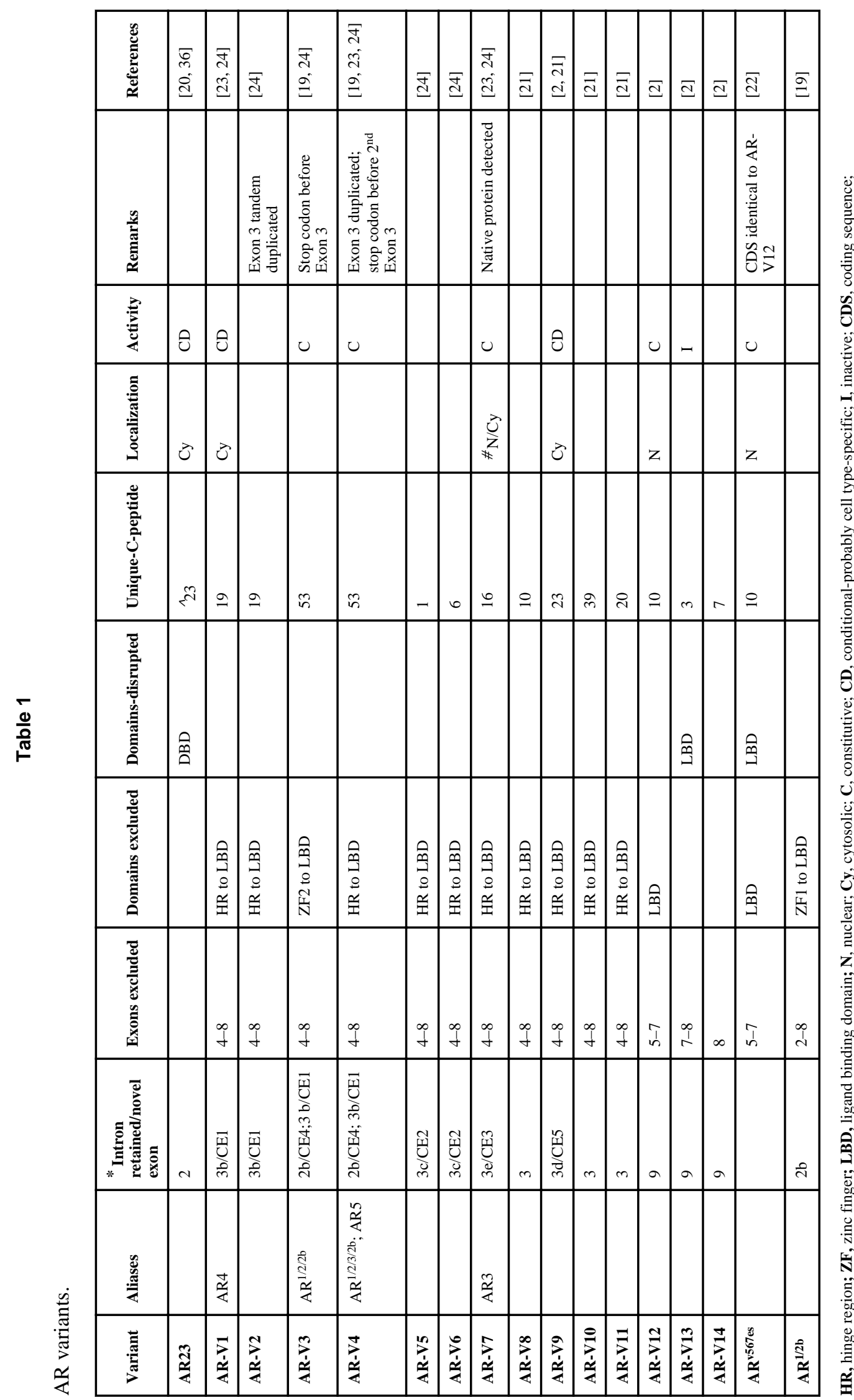

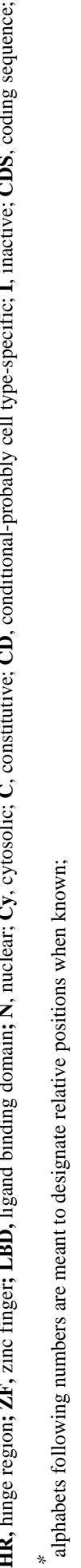




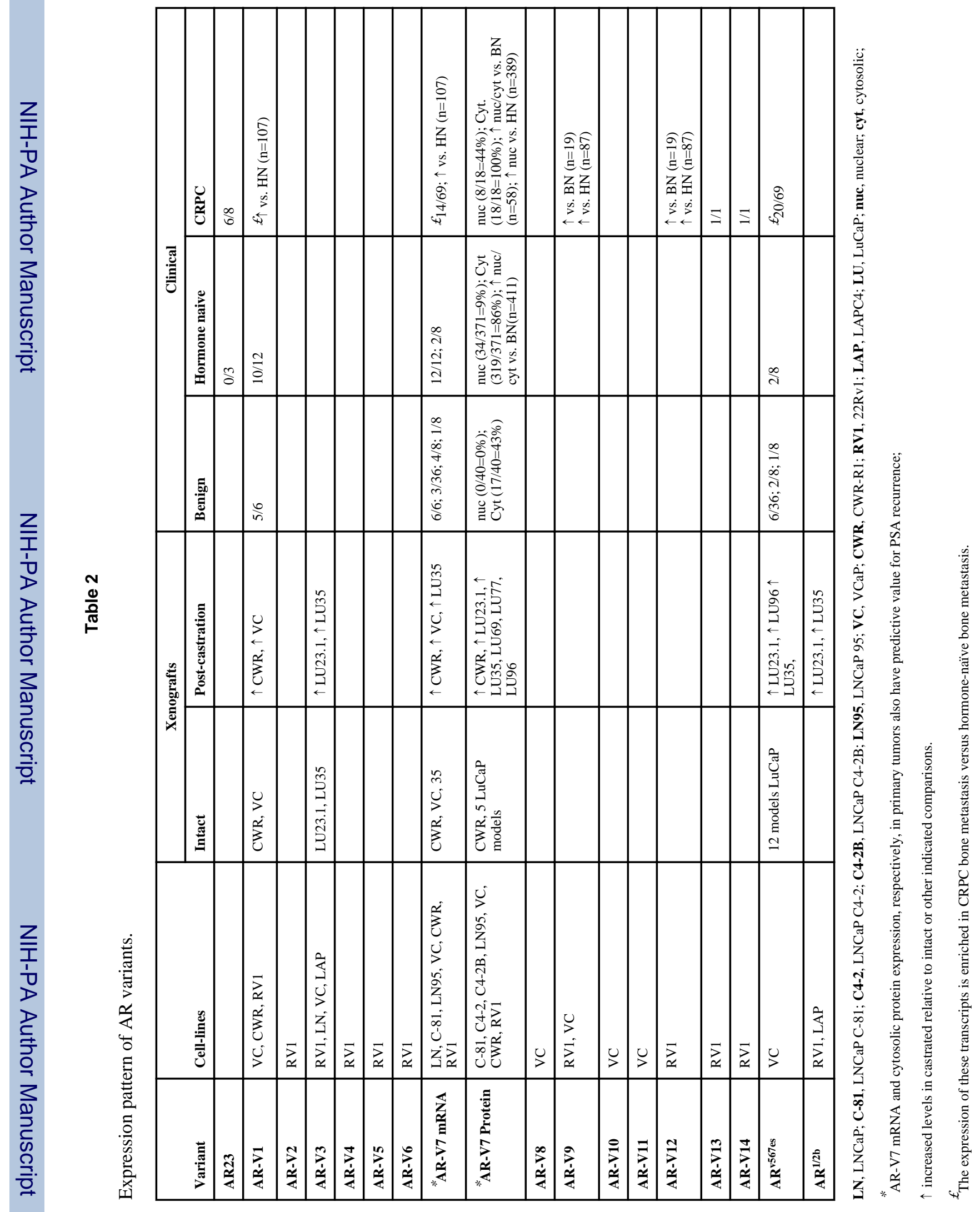

